\title{
Atrazine Sensing Chip Based on Molecularly Imprinted Polymer Layer
}

\author{
Izumi KuBo, ${ }^{\text {* }}$ Reo ShojI, ${ }^{a}$ Yusuke FuchiwaKi, ${ }^{a}$ and Hiroaki Suzuki ${ }^{\mathrm{b}}$
}

\author{
${ }^{a}$ Graduate School of Engineering, Soka University (Hachioji, Tokyo 192-8577 Japan) \\ bInstitute of material science, University of Tsukuba (Tsukuba, Ibaraki 305-8577 Japan)
}

Received February 23, 2008 ; Accepted July 9, 2008

\begin{abstract}
Atrazine is a widely used herbicide. The electrochemical sensing of atrazine was performed by the detection of its reductive current. In order to make the sensing system smaller and simpler, a gold electrode chip (detection area: $1.8 \times 0.6 \mathrm{~mm}$ ) was prepared and modified with an atrazine imprinted polymer layer, as the recognition element for atrazine. An atrazine sensing chip modified with molecularly imprinted polymer (MIP) layer was fabricated by spin-coating in this study. Using the atrazine-sensing chip, a cathodic current of atrazine was observed and the reductive current depended on the concentration of atrazine below $20 \mu \mathrm{M}$. The detection limit of the sensor was 50 nM (11 ppb). The atrazine-sensing chip was more selective to atrazine than other herbicides such as simazine, ametryn and MCC.
\end{abstract}

Key Words : Atrazine, Molecularly Imprinted Polymer (MIP), Gold, Cathodic Current, Layer

\section{Introduction}

Recently, artificial receptors typified by molecularly imprinted polymers (MIPs) have been intensively investigated. ${ }^{1,2}$ MIPs are in general more stable than enzymes and antibodies, so numerous reports have analyzed MIPbased sensors for the detection of theophyline, ${ }^{3)}$ proteins $^{4)}$ and 2,4-dichlorophenoxyacetic acid.5) A herbicide, atrazine is a candidate target for MIP-based sensor. Atrazine is one of the $s$-triazine herbicides which has recently been reported to be an endocrine disrupting chemical. ${ }^{6}$ ) MIPs for atrazine (atrazine-imprinted polymers) have been reported by several groups. Muldoon reported the application of MIP, which acted as a substitute for antibodies, to a competitive binding assay of atrazine using radioactively labeled ligand..$^{7}$ Another application of MIPs is solid-phase extraction for chromatography. In such applications, fine particles of MIPs were utilized as a solid phase to concentrate and separate atrazine from structurally analogous molecules. ${ }^{8,9}$ Piletsky et al. prepared atrazine-imprinted polymer from diethylaminoethyl methacrylate and ethylene glycol dimethacrylate on a glass filter ${ }^{10)}$ and utilized it to determine atrazine. MIPs were utilized as a molecular recognition membrane or as a layer in a chemical sensing system. The proposed applications are the combination of MIPs and transducers such as conductometry, ${ }^{11)}$ impedometry ${ }^{12)}$ or an acoustic device. ${ }^{13)}$ The principle of the above sensing devices is based on the physicochemical changes of the MIPs caused by the binding of atrazine.

Our research group has proposed an amperometric atrazine sensor using gold electrode covered with MIPs. ${ }^{14)}$ The detection principle was based on the molecular recognition by MIPs and electrochemical reduction of atrazine. ${ }^{15)}$ It was the first report of an amperometric atrazine sensor. The detection limit of the sensor was $1 \mu \mathrm{M}$, which is higher than atrazine concentration in the environmental waters. To improve the detection limit, a newly designed amperometric sensor was required.

In this study, a novel atrazine sensing chip was developed using a miniaturized gold electrode chip with MIP layer. The morphology of MIP layer on the electrode was observed by Atomic Force Microscopy (AFM). Our proposed sensing chip allowed highly sensitive detection of atrazine and repeated use.

\section{Experimental}

\section{1 Materials}

Atrazine [2-chloro-4 (ethylamino)-6-(isopropyl $\times \mathrm{sp}$ amino)-1,3,5-triazine] was obtained from Kanto Chemicals (Tokyo, Japan), simazine [2-chloro-4,6-(ethyl $\times$ sp amino)1,3,5-triazine], MCC (methyl-3,4-dichlorocarbanilate) and ametryn (2-ethylamimo-4-isopropylamino-6-methylthio1,3,5-triazine) were purchased from Wako Pure Chemical Industries, Ltd. (Osaka, Japan) as standard chemicals for testing herbicides. Wako Pure Chemical Industry supplied all other chemicals. $N, N$-dimethylformamide (DMF), methacrylic acid (MAA) and ethylene dimethacrylate (EDMA) were distilled prior to use in order to remove stabilizers. Other chemicals were laboratory grade and used without further purification.

\section{2 Preparation of atrazine sensing chip}

In this study, gold electrode chips were newly designed and fabricated as follows. The chips were prepared by sputtering gold onto a 3 inch glass wafer. Total active areas $(0.6 \times 1.8 \mathrm{~mm})$ were delineated with a polyimide layer (Fig. 1) and portioned into four areas by latticed polyimide lines. These latticed lines were designed to support the MIP layer because MIP is more adhesive to polyimide than to the surface of gold. On the surface of the gold electrode chip, atrazine-imprinted polymer was prepared as shown in Fig. 1 and served as an atrazine sensing chip. 
a

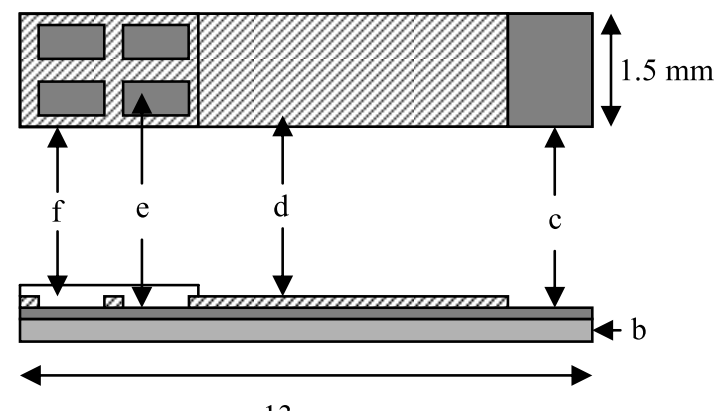

$13 \mathrm{~mm}$

Fig. 1 Schematic illustration of atrazine-sensing chip. MIP layer covers a sensing area of gold electrode latticed by a polyimide layer which supports MIP. a. Sensing area, b. Glass substrate, c. Contact area, d. polyimide layer, e. Gold electrode, f. MIP layer on sensing area.

The MIP layer was prepared on a gold chip according to a previously reported procedure. ${ }^{16)}$ The cleaned gold electrode chip was immersed overnight in ethanol/water (4:1, v/v) containing $53 \mu \mathrm{M}$ allyl mercaptan and $13 \mu \mathrm{M} 1$ butanethiol to immobilize the MIP layer covalently on the gold surface. The immersed electrode chip was dried with nitrogen gas.

The following cocktails of pre-polymer consisting of 8.1 $\mathrm{mg}$ atrazine, $30 \mu \mathrm{l} \mathrm{MAA}, 180 \mu \mathrm{l}$ EDMA and $4 \mathrm{mg} \alpha, \alpha^{\prime}-$ azobis (isobutyronitile) (AIBN) was purged with nitrogen gas for 10-20 sec. A droplet of pre-polymer cocktail was put onto the surface of the chip prepared above, and spun to control the thickness of the MIP layer by a spincoater at $800 \mathrm{rpm}$, for $3 \mathrm{sec}$. Immediately after spinning, polymerization was initiated by UV irradiation (CSL30BL, Cosmo Bio Co., Ltd., Tokyo, Japan) at $4{ }^{\circ} \mathrm{C}$, for 2 hrs. The polymer-coated electrode chip was immersed in methanol/acetic acid $(7: 3, \mathrm{v} / \mathrm{v})$ overnight to remove atrazine from the formed MIP layer. Prior to electrochemical measurements, the atrazine sensing chip was immersed in $0.14 \mathrm{M} \mathrm{LiCl} / \mathrm{DMF}$ for $1 \mathrm{hr}$, to introduce supporting electrolyte into the polymer layer.

\section{3 Electrochemical measurements}

A three-electrode cell was used in the electrochemical measurements. The prepared atrazine sensing chip, a platinum wire (BAS Inc.) and $\mathrm{Ag} / \mathrm{AgCl}(3 \mathrm{M} \mathrm{NaCl}$; BAS Inc., Tokyo, Japan) were used as a working electrode, an auxiliary electrode and a reference electrode, respectively. Electrochemical measurements were carried out in a glass vial containing $10 \mathrm{~mL}$ of electrolyte solution $(0.1 \mathrm{M}$ $\mathrm{KCl}$ ) at room temperature, $\mathrm{pH} 3.0$, according to a previously reported procedure. ${ }^{14,16)}$ Atrazine was detected by cyclic voltammetry under the above setup.

\section{Results and Discussion}

\section{1 AFM image of a MIP layer}

There are no reports on the morphology of an atrazine MIP membrane or a layer observed by AFM. We considered that MIPs are porous and that a template molecule such as atrazine diffuses into MIP through pores and binds to imprinted sites. In order to ensure such mor-

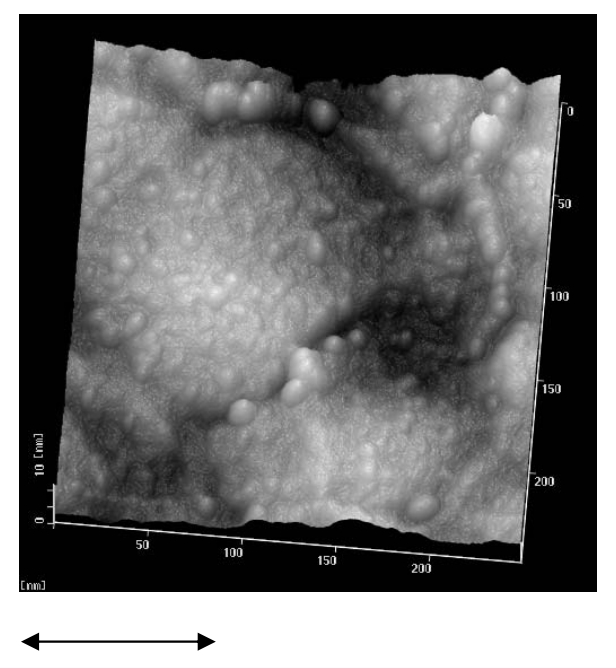

$100 \mathrm{~nm}$

a. Bare gold chip

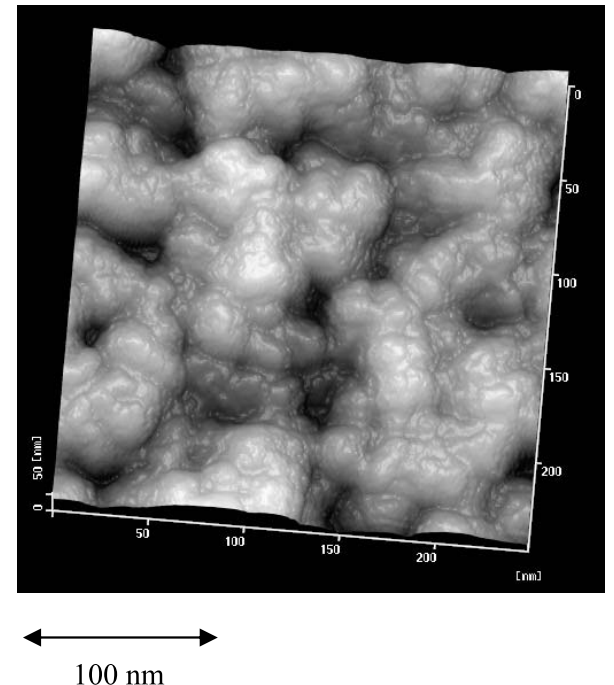

$100 \mathrm{~nm}$

b. Atrazine sensing chip

Fig. 2 AFM images of bare gold chip and MIP layer on the chip. Images of $10 \mathrm{~nm} \times 250 \mathrm{~nm} \times 250 \mathrm{~nm}$. a. Bare gold electrode chip which was prepared on glass substrate by sputtering of gold, b. Atrazine-imprinted polymer layer was prepared on the bare gold electrode chip.

phology, the MIP layer of the atrazine-sensing chip was observed by AFM to investigate the morphology of the MIP layer immobilized on the gold chip electrode surface. AFM images are shown in Fig. 2. The surface of the chip modified with imprinted receptor was clearly different from that of a bare gold chip. As for the bare gold chip, the surface was covered with sputtered gold particles whose diameter ranged from 5 to $10 \mathrm{~nm}$ (Fig. 2 (a)). After the MIP layer was prepared on the surface of the sensing chip, the surface was completely covered with densely packed particle-like structure, whose diameter was $10-20 \mathrm{~nm}$, and not so porous (Fig. 2 (b)). This structure suggests that template molecules will not easily diffuse to the surface of the electrode. Therefore a 
thinner layer will result in easier diffusion of atrazine and higher sensitivity.

\section{2 Effect of spin-coating on Atrazine response}

At first we examined the cyclic voltammogram of atrazine with the use of the prepared atrazine sensing chip. The cathodic current of atrazine, which became greater at a lower potential and reached a peak at around $-800 \mathrm{mV}$, was proportional to the concentration of atrazine. Then the cathodic current difference between with and without atrazine at $-800 \mathrm{mV}$ was measured as a reductive current of atrazine, hereafter. These results were consistent with our previous studies using a different shape of electrode..$^{14)}$

We measured atrazine concentration using the chip with a spin-coated MIP layer, and examined the relation between the concentration of atrazine and the difference in cathodic current. The cathodic current response to 10 $\mu \mathrm{M}$, which was 1.82 times greater than that of $5 \mu \mathrm{M}$, depended on the concentration of atrazine. The thickness of the spin-coated MIP layer was $35 \pm 3.5 \mu \mathrm{m}$ and that of the MIP layer without spin-coating was $65 \pm 12.1$ $\mu \mathrm{m}$. The current response of the atrazine-sensing chip prepared without spin-coating was $82 \%$ of that with a spin-coated MIP layer. Spin-coating improved the current response.

\section{3 Atrazine-sensing chip response}

The response of atrazine concentration range of 0.5-20 $\mu \mathrm{M}$ was measured. The reductive current of atrazine depended on the concentration of atrazine. The relation between the reductive current response and the concentration of atrazine is shown in Fig. 3. A linear relationship between atrazine concentration and the reductive current was observed at the range of $0.5-15 \mu \mathrm{M}$ of atrazine.

We then applied the atrazine-sensing chip to determine the lower concentration of atrazine. The result is shown in Fig. 3 (inset). At lower concentrations the cathodic current responses to atrazine depended on the concentration, and the detection limit was $50 \mathrm{nM}$. The cathodic current response was $-270 \mathrm{nA}$ to $50 \mathrm{nM}$ of

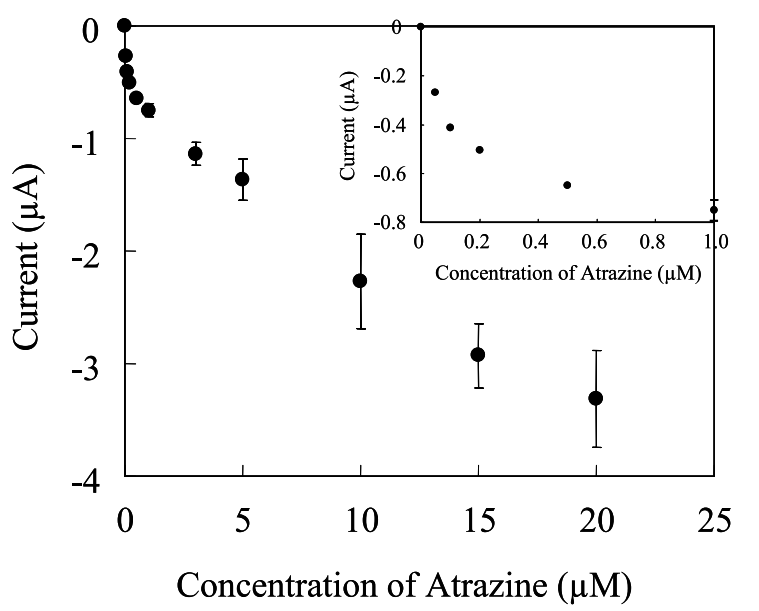

Fig. 3 Calibration plot of atrazine with atrazine-sensing chip. Current value is the difference at $-800 \mathrm{mV}$ in the presence and absence of atrazine. atrazine. In this work we improved the detection limit over our previous report because of the thinner layer of MIP by spin-coating.

\section{4 Selectivity of atrazine-sensing chip}

The response of the atrazine-sensing chip to other herbicides was examined. Simazine, ametryn and MCC were compared. Simazine and ametryn belong to the triazine herbicides, and MCC is used as a pesticide, which does not have a triazine ring but chlorine. These chemical substances showed cathodic responses as atrazine on a bare gold electrode chip. Then the difference in current between these substances was compared using a bare gold electrode chip and an MIP-modified electrode. The responses of each substance $(5 \mu \mathrm{M})$ were compared to the response to atrazine with the MIP-modified electrode chip, shown in Fig. 4. The response to atrazine did not change markedly by modifying the gold electrode chip

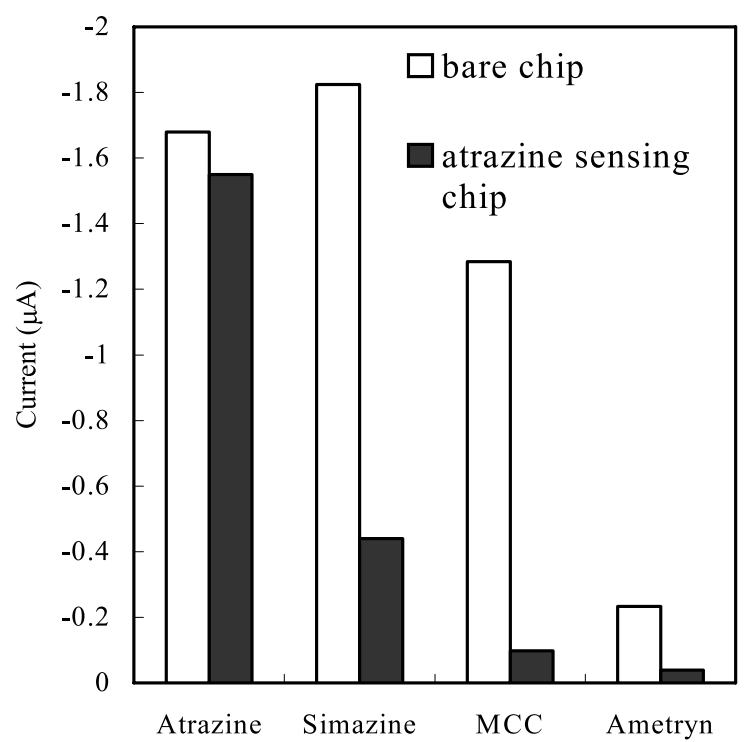

Fig. 4 Comparison of the responses of the atrazine sensing chip and a bare gold chip to various herbicides. Current response (bare gold chip and atrazine sensing chip) of each substances $(5 \mu \mathrm{M})$ is shown.

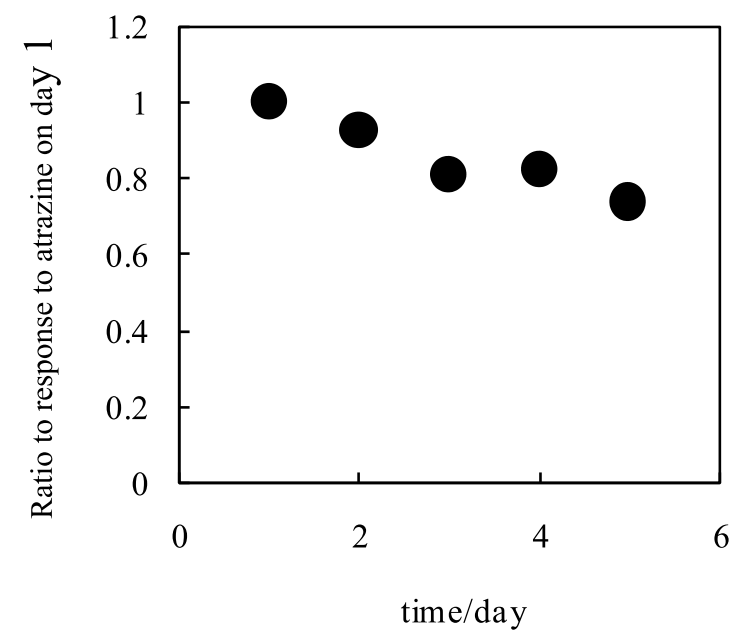

Fig. 5 Reusability of atrazine sensing chip. Ratio of the each day response to the day 1 (Atrazine $5 \mu \mathrm{M}$ ). 
with the atrazine-imprinted polymer layer. The response of the gold electrode chip to simazine and MCC was not so different from that to atrazine. On the other hand the response of the atrazine-sensing chip to simazine and MCC was rather smaller than that to atrazine. Ametryn showed the smallest response even with the gold electrode chip among the examined substances, and with the atrazine-sensing chip, little response was observed. From this result, the atrazine-sensing chip prepared in this study was shown to be more selective to atrazine.

\section{5 Reusability}

Finally, reusability was tested. Five $\mu \mathrm{M}$ of atrazine was measured day by day. The sensing chip could be used to determine atrazine over 5 days (Fig. 5), maintaining $80 \%$ of its initial response. The stability and adhesive property of the receptor layer were improved with the latticed polyimide structure of the gold electrode chip prepared in this study.

\section{Conclusion}

In this study, an atrazine-sensing chip with an MIP layer on gold electrode chip was developed. The atrazine-imprinted polymer was used as a recognition element. The reductive current of atrazine was measured using the atrazine-sensing chip by cyclic voltammetry. The linear relationship between atrazine concentration and the reductive current was observed in the range of 0.5 to $15 \mu \mathrm{M}$. The minimum detectable concentration of atrazine was $50 \mathrm{nM}(11 \mathrm{ppb})$. Our proposed sensing chip allows highly sensitive detection of atrazine and repeated use.

\section{Acknowledgment}

This research was supported by a grant from the Ministry of Education, Culture, Sports and Technology and the Kato science foundation. The authors also grate- fully thank Dr. Masaji Shigeno of Seiko Instruments for assistance of AFM observation.

\section{References}

1) K. Mosbach, Trends Biochem. Sci., 19, 9 (1994).

2) K. J. Shea, Trends Polym. Sci., 2, 166 (1994).

3) Y. Yoshimi, R. Ohdaira, C. Iiyama, and K. Sakai, Sens. Actuators B, 73, 49 (2001).

4) A. Bossi, S. A. Piletsky, E. V. Piletska, P. G. Righetti, and A. P. F. Turner, Anal. Chem., 73, 5281 (2001).

5) H. Peng, F. Yin, A. Zhou, and S. Yao, Anal. Lett., 35, 435. (2002).

6) P. M. Vonier, D. A. Crain, J. A. McLachlan, L. J. Gullette Jr, and S. F. Arnold, Environ. Health Perspect., 104, 1318 (1996).

7) M. T. Muldoon and L. H. Stanker, J. Agrc. Food Chem., 43, 1424 (1995).

8) J. Matsui, Y. Miyoshi, O. Doblhoff-Dier, and T. Takeuchi, Anal. Chem., 67, 4404 (1995).

9) T. A. Sergeyeva, H. Matuschewski, S. A. Piletsky, J. Bendig, U. Schedler, and M. Ulbricht, J. Chromatography A, 907, 89 (2001).

10) S. A. Piletsky, E. V. Piletskaya, A. V. Elgersma, K. Yano, and I. Karube, Biosens. Bioelectron., 10, 969 (1995).

11) T. A. Sergeyeva, S. A. Piletsky, A. A. Brovko, E. A. Slinchenko, L. M. Sergeeva, and A. V. El' skaya, Anal. Chim. Acta, 392, 105 (1999).

12) T. Panasyuk-Delaney, V. M. Mirsky, M. Ulbrich, and O. S. Wolfbeis, Anal. Chim. Acta, 435, 157 (2001).

13) C. Luo, M. Liu, Y. Mo, J. Qu, and Y. Feng, Anal. Chim. Acta, 428, 143 (2001).

14) R. Shoji, T. Takeuchi, and I. Kubo, Anal. Chem., 75, 4882 (2003).

15) L. Pospissil, R. Trskova, R. Fuoco, and M. P. Colombini, J. Electroanal. Chem., 395, 189 (1995).

16) R. Shoji, T. Takeuchi, H. Suzuki, and I. Kubo, Bunseki Kagaku, 52, 1141 (2003). 